\title{
A Study on an Air-Jet Loom with Substreams Added
}

\author{
Part 5: Analogous Experiment of Flow \\ By Minoru Uno, Member, TMSJ \\ Kyoto Institute of Technology, Kyoto \\ Based on the Journal of The Textile Machinery Society of Japan, Transactions, Vol. 25, No. 11, T224-231 (1972)
}

\begin{abstract}
In order to visualize the flow pattern on an air jet loom, the electrolytic tank analogue technique was studied to get the following results:

1) In case of an axisymmetric flow of a perfect fluid, the direct wedgetank analogue can be utilized easily and accurately for plotting of equipotentials, and the orthogonal hyperbolic tank analogue for plotting of stream lines.

2) The analogue technique mentioned above is applied to the flow in a nozzle to clarify the flow pattern there and to know the effect of the yarn guide length on the flow.

3) The same technique is also applied to the shed to show the rapid air flow against or through the slay. If the substreams are added, this flow is somewhat lessened, resulting in improvement of axial stream.
\end{abstract}

KEY WORDS: LOOMS, AIF JET LOOMS, FLOW (FLUIDS), VELOCITY, DISTRISBUTION, POTENTIAL (ELECTRICAL), TANKS, NOZZLES, SHED (WEAVING), DENTS

\section{Introdution}

As the weft propulsion on an air-jet loom is performed by rapid air stream in the yarn-gushing-out nozzle or along the shed, it is very important to visualize the stream lines in order to get clear understanding of air action there. Thus Pitot-tubes or hot-wire anemometers are frequently used to measure the local air velocity. But the space in the shed is too small and warps should be separated sideways to measure the velocity conveniently with such devices. To overcome these defects, a large model can be considered, but it needs too much air consumption and in some cases cannot be carried out.

Filtered smoke from burning rotten wood or from the chemical titanium tetrachloride can be used to take the flow photograph, but to such a high-dispersing flow as jet enough smoke density is hardly obtained to get clear pictures. Especially, the latter substance has the defect corrosive to many materials.

For simple experiment, saw-dust or aluminium flakes are mixed in jet-air, but the density is too different from that of the air to get quantitative result. ${ }^{[1]}$
So, in this report, the electrolytic tank analogue technique is utilized to visualize the flow pattern in the main nozzle and the shed on air-jet looms, after assuming air to be an ideal inviscid gas. The research in the textile domain by the e'ectrolytic tank technique is very few, excepting the study by Denton ${ }^{[2]}$ on the dye flow through the yarnwound package. But he drew stream lines by hand orthogonally to the equi-pressure lines, including some errors speaking rigorously. In this paper, Beaver's method ${ }^{[3]}$ is applied which gives the correct solution for stream lines of the axisymmetric flow of a perfect fluid, and some results are reported after using two kinds of electrolytic tanks.

\section{Basis of Electrolytic Tanks}

The Laplacian field can generally be considered to be a flow, having no curls, no sources and no sinks, in that domain, ending the stream lines only on the boundary, composed of incompressible fluid. Then, in the two-dimensional flow in $x-z$ plane, the velocity potential $\phi$ and the stream function $\Psi$ are orthogonal to each other, giving the 


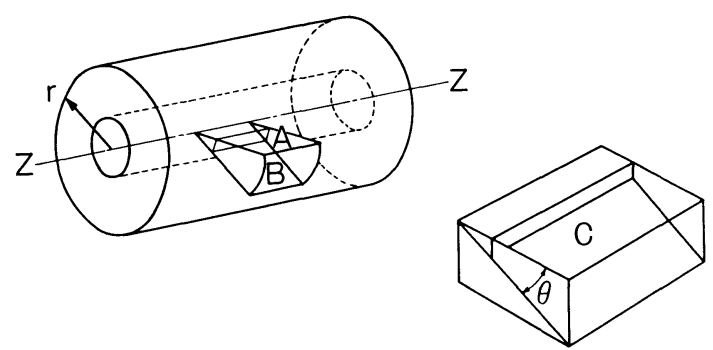

Fig. 1 Electrolytic tank for an axisymmetric flow

velocities $u, v$ in the $x, z$ directions respectively as

$$
\left.\begin{array}{l}
u=\frac{\partial \phi}{\partial x}=\frac{\partial \Psi}{\partial z} \\
v=\frac{\partial \phi}{\partial z}=-\frac{\partial \Psi}{\partial x}
\end{array}\right\}
$$

This relation is identical to that between electric current and electric voltage. So, to obtain the flow pattern, can be utilized the electrolytic tank method which gives such lines as $\phi=$ const, $\Psi=$ const, after putting the elctrodes in a water tank having constant depth. In this case, the same flow patterns are obtained even if the electrodes and the insulating walls are exchanged between them.

By the extension of this idea to the axisymmetric flow, Barkhausen $^{[4]}$ showed the possibility of obtaining the velocity potential by the wedge-type electrolytic tank $\mathrm{C}$ shown in Fig. 1, in which the circular cylinder $(z, r)$ represents the axisymmetric flow and the wedge is made of planes $\mathrm{A}$ and $\mathrm{B}$, both containing the $z$-axis. In practice, a shallow water tank is slightly tilted and the probe is moved just below the horizontal water surface. If the wedge angle $\theta$ is as small as $5-10^{\circ}$, vertical walls can be used with little error instead of cylindrical walls. But the exchange of the electrode and the insulating wall does not lead to the $\operatorname{correct} \Psi$, because the intervals between equipotential curves should be adjusted inversely as the radius increases. In other words Laplace's equation for the usual cylindrical co-ordinates $(r, \theta, z)$ is

$$
\frac{\partial^{2} \Omega}{\partial r^{2}}+\frac{1}{r} \frac{\partial \Omega \Omega}{\partial r}+\frac{\partial^{2} \Omega}{\partial z^{2}}=0
$$

whereas, the stream function is, different from the above,

$$
\frac{\partial^{2} \Psi}{\partial r^{2}}-\frac{1}{r}-\frac{\partial \Psi}{\partial r}+\frac{\partial^{2} \Psi}{\partial z^{2}}=0
$$

But if such a transformation is done as

$$
\left.\begin{array}{l}
x y= \pm p^{2} \\
x^{2}-y^{2}= \pm q^{2} \\
z=z
\end{array}\right\}
$$

and is introduced a new function $\Theta$ which satisfies the followig boundary condition,

$$
\left.\begin{array}{c}
\frac{\partial \Theta}{\partial p}=0 \\
\frac{\partial^{2} \Theta}{\partial p^{2}}=0
\end{array}\right\}
$$

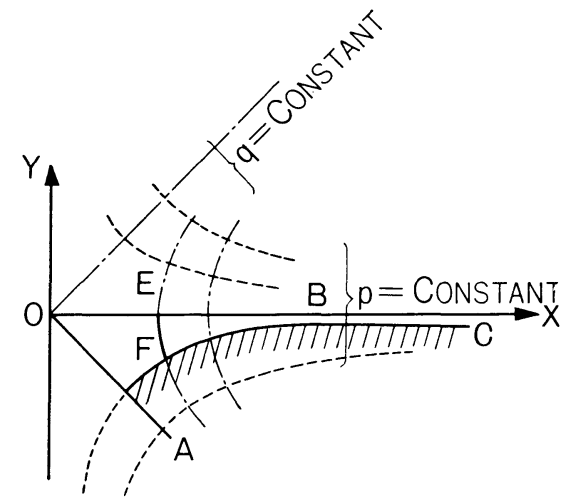

Fig. $2 p, q$ curves

Laplace's equation for $\Theta$ is, on the surface $p=0$,

$$
\frac{\partial^{2} \Theta}{\partial q^{2}}-\frac{1}{q} \frac{\partial \Theta}{\partial q}+\frac{\partial^{2} \Theta}{\partial z^{2}}=0
$$

This is identical, as Beaver showed, to the stream function (3). Eq. (4) defines these orthogonal hyperbolic curves as shown in Fig. 2, in which the symmetric axis is represented by $45^{\circ}$ plane OA $(q=0)$, the surface of electrolyte by plane OB $(p=0)$, the bottom by hyperbolic surface CF ( $p=$ const.). The electrode EF is orthognonal to both surfaces $\mathrm{OB}$ and $\mathrm{CF}$, and is hyperbolic too.

\section{Construction of Electrolytic Tank}

\subsection{Tank for Velocity Potential}

The water tank used for observing the velocity potential is shown in Fig. 3. It has the outer dimensions of $100 \times$ $60 \times 60 \mathrm{~cm}$, is made of transparent acrylic plates (1), is tilted about $8^{\circ}$ against horizon, and filled with tap water. Shore line AB corresponds to the axis of a nozzle.

To visualize the flow pattern in the nozzle into which the compressed air is blown obliquely but symmetrically, a cavity (7) made of acrylic plate is formed on the nozzle inner wall (8) made also of acrylic plate. Yarn guide pipe is represented by acrylic plate (5), parallel to the shore line.

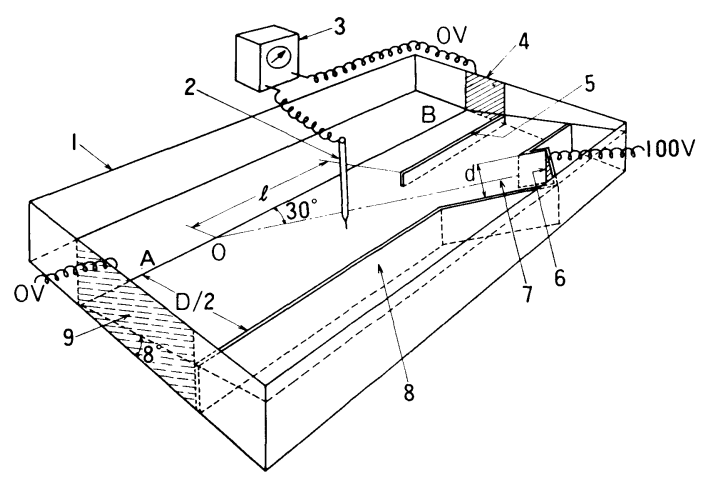

Fig. 3 Electrolytic tank for velocity potential 


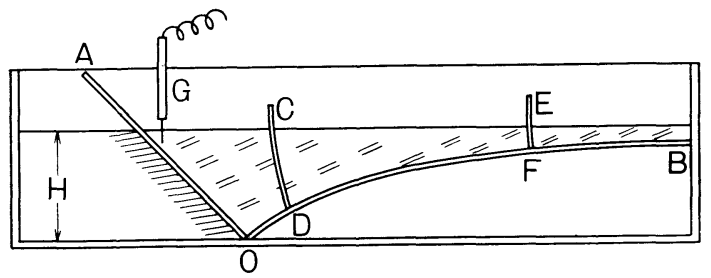

Fig. 4 Hyperbolic tank

The cavity width $d$ is determined by the following formula to conform to the previous experiment,

$$
d=\frac{2 \pi d_{0}^{2}}{\pi D_{0}}
$$

This is because, in Part 4 the main nozzle $\left(I D=D_{0}\right)$ was fed with air through two small pipes $\left(I D=d_{0}\right)$, but in the analogue experiment the configuration corresponds to the air supply through a ring-like slit arround the axis.

$0 \mathrm{~V}$ is put to electrodes (4), (9) to analogize the open air, and $100 \mathrm{~V}$ to electrode (6) to analogize the supply air. $D$ and $d$ in Fig. 3 are $40 \mathrm{~cm}$ and $11.6 \mathrm{~cm}$ respectively, and probe (2) is moved to read the electric voltage with voltmeter (3).

\subsection{Tank for Stream Function}

The tank is now set horizontal, and the water depth $H$ shown in Fig. 4 is made $6 \mathrm{~cm}$. Hyperbolic plates OB (vinyl),

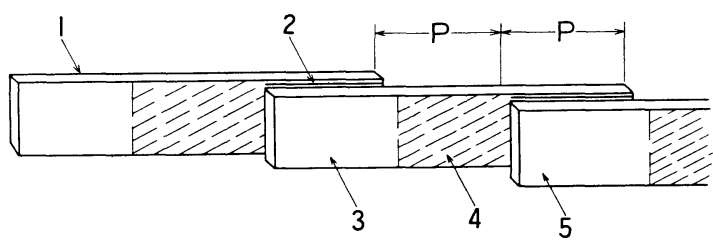

Fig. 5 Shed-analogizing electrode

$\mathrm{CD}(A l), \mathrm{EF}(A l)$ make the bottom, the guide-tube wall and the nozzle outside-wall respectively. Electrode $\mathrm{OA}$ is inclined to the water surface by $45^{\circ}$, and corresponds to the axis of symmetry. Probe $\mathrm{G}$ should scan as shallow as possible to satisfy the condition $p=0$.

\subsection{Tank for Shed}

Air flows away through dents of the slay. To analogize such heterogeneous wall, conductive pins on insulating walls are suggested by Warmoltz. ${ }^{[6]}$ But as it was too tedious to adjust many pins, such aluminium plate arrangement is adopted as shown in Fig. 5, in which the adhesive but electrically insulating paper (2) or (4) is inserted between plates (1), (3), or (5).... Pitch P, the distance which determines the voltage drop between two adjacent constant voltage parts, should be adjusted according to each air leakage rate of the slay used on air jet looms.

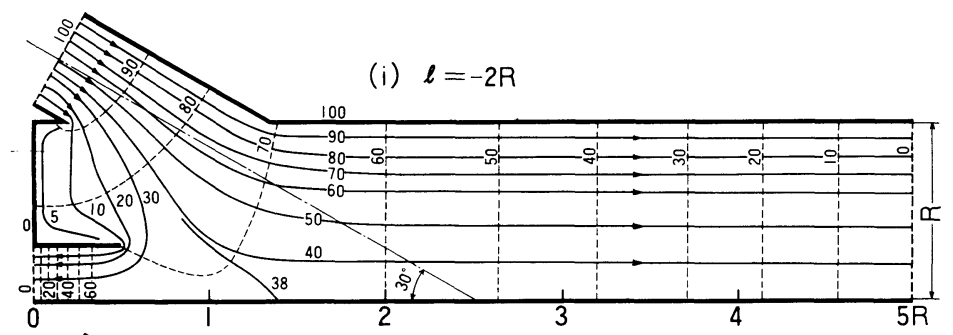

(ii) $\ell=0$
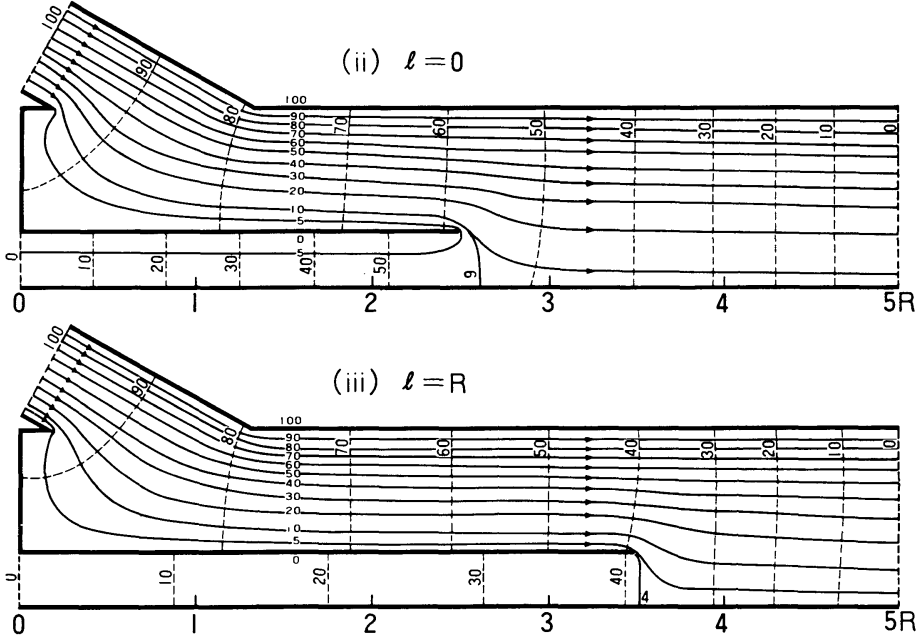

Fig. 6 Effect of the guide-pipe-tip position on the flow in a nozzle $\left(L / R=5.30^{\circ}\right.$ inclination $)$ 


\section{Experimental Results}

\subsection{Flow in Nozzles}

The flow pattern in the cylindrical nozzle, having a yarn guide pipe on its axis, having the radius $R$ and the length $L=5 R$, is shown in Fig. 6 when air is injected axisymmetrically and inclined $30^{\circ}$ against the nozzle axis toward such a geometrical air-meeting point, $2.5 R$ deep from the exit of the nozzle. Only the upper half sections are shown in Fig. 6, in which the full lines are stream lines observed in the hyperbolic water tank when the nozzle inner wall is charged with $100 \mathrm{~V}$ and the guide-pipe wall with $0 \mathrm{~V}$. The electrode corresponding to the symmetrical axis is not charged positively, but is left charged of itself. Broken lines are the equipotential lines obtained with the wedge-type tank, of which the voltage is given as explained in Section 3.1. Digits in the figure show the local voltages, from which the flow rate can be deduced. As for the yarn guide pipe, about $38 \%$ of the total fed-in air-volume flows out backward through it when, as shown in Fig. 6 (i), $l=-2 \mathrm{R}$ where $l$ is the distance between the guide-pipe tip and the injected-air meeting point, taking the positive sign when the pipe tip is nearer to the nozzle exit. When $l=-2.5 R$, that is, when there were no guide pipe, $68 \%$ of the original fed volume flows away backward, as shown in Fig. 7. But as the guide pipe is made long, the adverse flow diminishes, and reaches $9 \%$ when $l=0$, or $4 \%$ when $l=R$. Fig. 6 (ii) and (iii) show these cases. The result agrees well with that obtained with Pitot-tubes and reported in Part 4. ${ }^{[5]}$

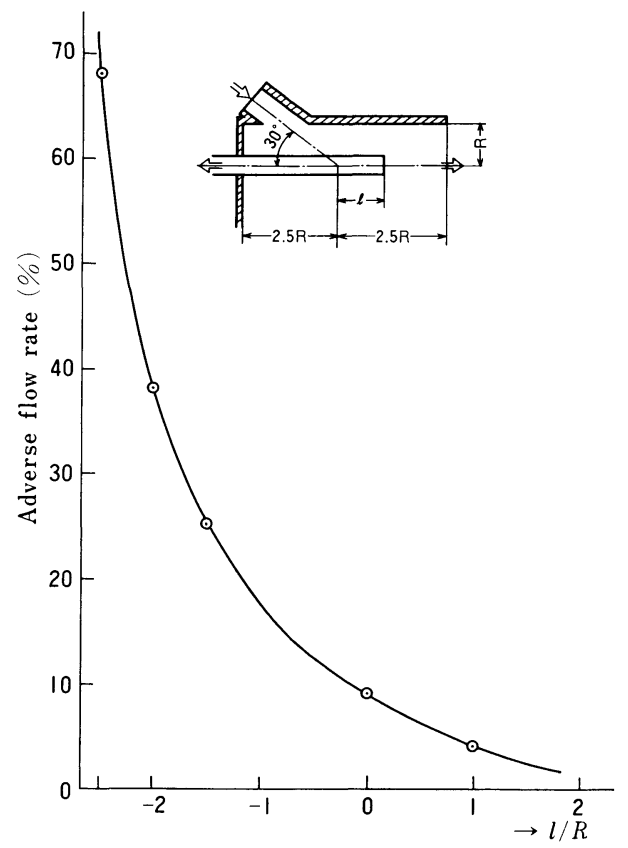

Fig. 7 Relation ketween adverse flow and guide-pipetip position $l$.

\subsection{Flow in Sheds}

The flow pattern in the shed when no air leaks through warps by dint of suitable covers set outside of warps is obtained by the electrolytic analogue technique and shown in Fig. 8.
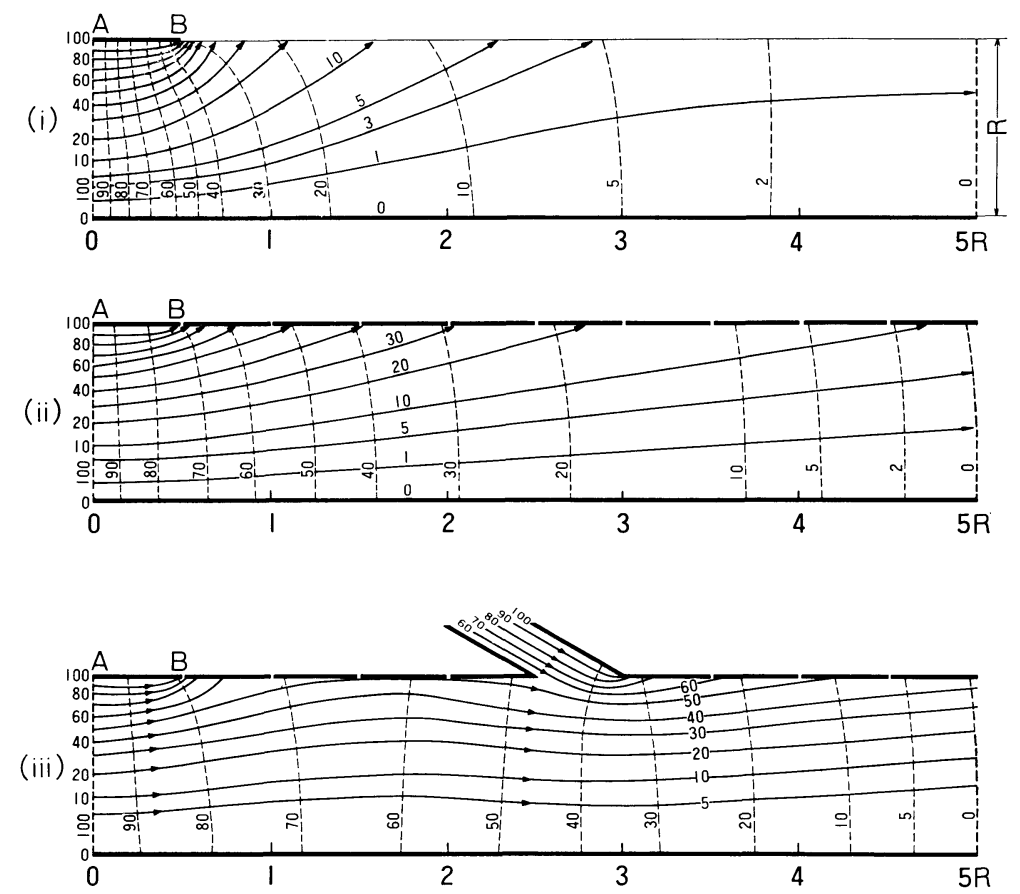

Fig. 8 Flows in sheds. (i) No dents. (ii) With dents. (iii) With substreams added. 

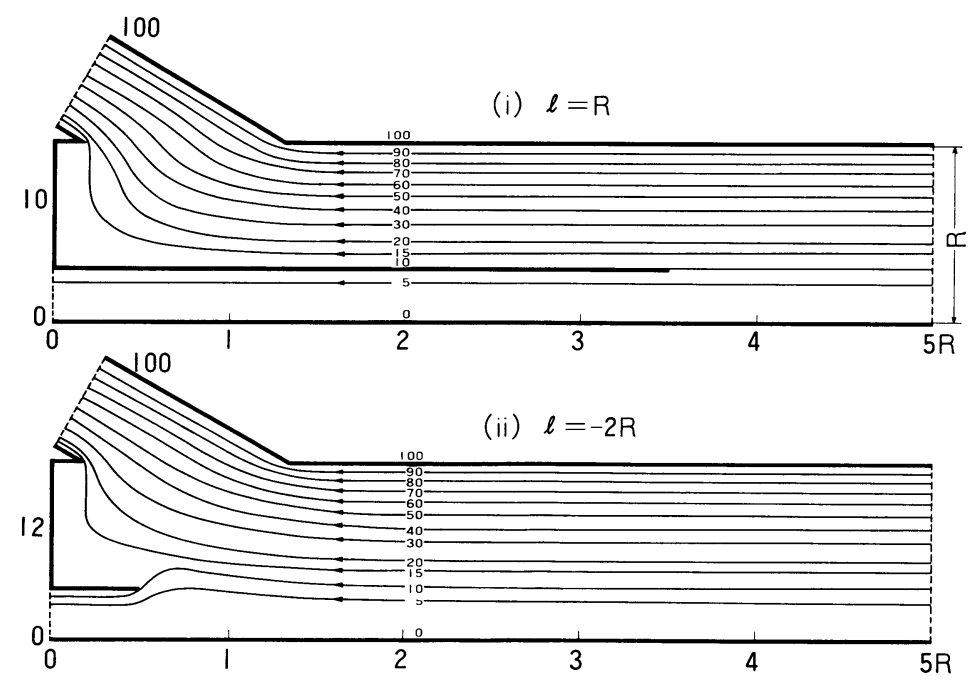

Fig. 9 Adverse flow into a nozzle

Fig. 8 (i) is the case in which the reed has no dents. As air can flow freely through the back-side of the shed, stream lines change their directions rapidly, and the tendency is intense near the left entrance to which air is blown in towards right. Almost half of the air fed in flows out of the shed within $0.1 R$ from $\mathrm{AB}$ with which the shed is covered at the dent position, where $R$ is the shed depth. Fig. 8(ii) shows the flow when the shed has dents and their pitch $P$ defined in Fig. 5 is $0.5 R$.

Although stream lines are flatter than those in Fig. 8(i), about $50 \%$ of air leaks out within $0.6 R$ from $\mathrm{AB}$. These indicate that air does not flow parallel to the shed axis, that it flows violently toward dents. This agreees well with that reported in Part 3. ${ }^{[7]}$

In order to decrease the air-leakage and accelerate the air in the shed, it is usual to add substreams from outside of warps. But in this analogue technique no voltage can be applied on the wedge planes. So the flow in Fig. 8 (iii) indicates the case in which sub-air is blown in through dents from a slit, $0.5 R$ wide and $30^{\circ}$ inclined. The $50 \%$ stream line just touches the dent and again comes in the shed due to the air injection. If the slit is set nearer to cover $\mathrm{AB}$, this hopeful tendency will be promoted. But about $30-40 \%$ of air leaks out already within $0.2 R$ from cover $\mathrm{AB}$, so the merit to do so will not be so large. There should be some compromise between the air consumption due to sub-air injection, and the longer weft flight due to powerful air supply.

\subsection{Other Examples}

This analogue technique can be applied to many cases for visualizing the flow pattern. One example is illustrated in Fig. 9 in which air is blown in adversely through the exit of the nozzle. This time the $45^{\circ}$ electrode corresponding to the symmetric axis is charged with $0 \mathrm{~V}$, the nozzle inner

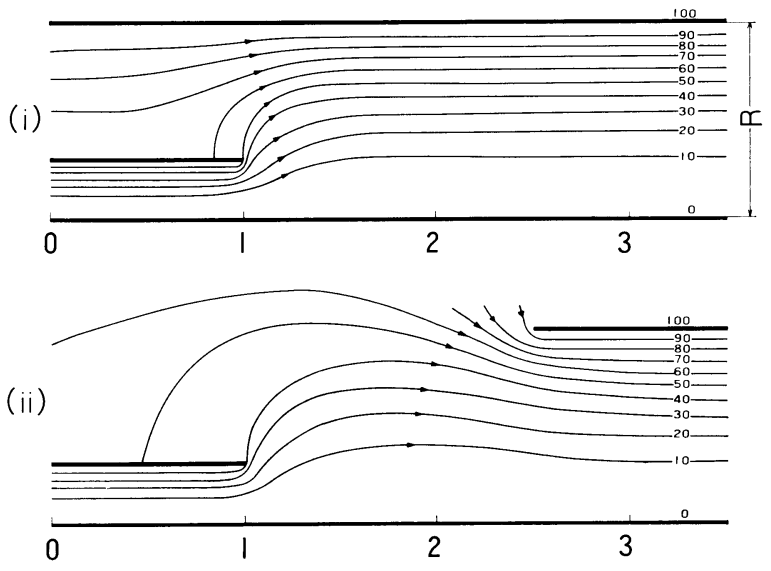

Fig. 10 Flows in and out of ducted nozzles

wall with $100 \mathrm{~V}$, and the guide tube is left charged of itself. There is only little difference between $l=R$ and $l=-2 R$, and only 10 or $12 \%$ of the air flows out through the guide pipe in contrast to $38 \%$ or more leakage in normal gushing state.

Fig. 10 shows the flow in and out of the ducted nozzles. The rigorous theoretical solution can hardly be obtained with such ducted nozzles. But the analogue tank technique can easily and quickly show the streamlines.

\section{Discussion}

\subsection{Solution of Axisymmetric Flow}

By availing of Beaver's method, correct stream lines can be obtained in axisymmetric flow, which is frequently observed in textile industry, such as through cones, cheeses, or fabric rolls. If once stream function $\Psi$ is determined, the equipotential lines can easily be calculated as follows. If $r$ 
is the distance between arbitrary stream line and the symmetric axis (z-axis), the flow velocity $v$ along $\mathrm{z}$ axis is, due to the constancy of the volumetric flow rate along that stream line;

$$
\pi r^{2} v=\Psi=\text { const }
$$

Then, from eq.(1),

$$
\phi=\int v d z=c \int \frac{d z}{r^{2}}
$$

where $c$ is a suitable constant. The right hand side of eq. (8) can easily be integrated if the stream line has been obtained. The broken lines showing $\phi=$ constant in Fig. 8 are drawn by this method without using the wedge-type tank.

\subsection{Flow of a Real Fluid}

When the solution of the mathematical equations relating to two characteristics of the flow is not easy to find, either by mathematical analysis or by direct experiment, it is sometimes possible to solve such problems much more easily by an experimental investigation in a corresponding field of analogous physical phenomena which themselves obey the same mathematical equations and boundary conditions.

Such an analogous experiment can also be applied to a field having curls, sinks or sources. But in case of an axisymmetric flow obeying Laplacian equation, the fluid should be incompressible and inviscid. Therefore Navier Stoke's equation cannot be satisfied.

So the method discussed here cannot solve a free jet flow having the core in the center and showing high pressure drop, which is heavily affected by the viscosity of the fluid. Likewise, the optimum inclination angle of the subnozzles against the shed axis cannot be deduced by the electrolylic tank technique. But in a real fluid, the flow is too unstable by eddies ${ }^{[9]}$ to get clear stream lines. The intermittent gushing of jets exaggerates that tendency. So, the visualization of flow patterns of an ideal fluid will be very beneficial to the first step to understand the behavior of a real fluid.

\subsection{Slay Dent}

There are very few data on the leakage of air through dents of the slay. It will be influenced by the structure of the slay, its dent density or by the loom speed. So in this paper, only the case in which $P=0.5 R$ is studied. Especially if the dent had $\sqsupset$ shape $^{[10]}$, it would show very peculiar aerodynamic character and would leave much room to be studied.

\section{Conclusion}

The electrolytic tank analogue technique has been studied to get the velocity potential and the stream function of an axisymmetric flow. The results are:

i) In case of an axisymmetric flow of a perfect fluid, the direct wedge tank analogue can be utilized easily and accurately for plotting of equipotentials, and the orthogonnal hyperbolic tank analogue for plotting of stream lines. ii) The analogue technique mentioned above is applied successfully to the flow in a nozzle, having a yarn guide pipe in the center and a ring-like slit on the surrounding wall. Clear visualization of the flow pattern is obtained and the effect of the yarn guide length on the flow can also be deuduced.

iii) The same technique is also applied to the shed to show the violent air flow against or through the slay dents. If substreams are added along the shed, this unfavourable flow can be decreased a little, resulting in improvement of axial stream.

iv) The electrolytic tank technique can only be applied to a inviscid flow following Laplacian equation, and is difficult to treat jets. But as there are many axisymmetrical packages in textiles, the wedge-type tank and the hyperbolic tank will be fruitfully applied in this industry.

Thanks are due to Miss Hisae Nakanisi who helped the experiment.

\section{Literature Cited}

[1] Asanuma, J. Japan. Soc. Mech. Eng., 72, p. 1370 (1969)

[2] Denton, M. J.; J. Text. Inst., 50, T521 (1959)

[3] Beaver, W. L.; Appl. Phys., 28, p.579 (1957)

[4] Barkhausen, von H., Bruck, J. von; Electrotech. Z., 54,175 (1933)

[5] Uno; J. Text. Mach. Soc. Japan. (Eng.ed), 18, p. 114 (1972)

[6] Vitkovitch, D.; Field Analysis, p. 215, D. Van Nostrand Co., 1966, London

[7] Uno; J. Tech. Mach. Soc. Japan. (Eng.ed), 18, p. 106 (1972)

[8] For instance, Pai, S. I. ; Fluid Dynamics of Jets, D. Van Nostrand Co., 1954, New York

[9] For instance, Curtet, R., Ricou, F. P., Trans. ASME. Ser. D. 86, 765 (1964) or Barchilon, M., Curtet,, R., ibid, 777.

[10] For instance, the slay made by De Strake. 